\title{
3D Reconstruction of the Rotational Axis in Fission Neutron Tomography
}

\author{
Oliver Kalthoff ${ }^{1, a^{*}}$, Thomas Bücherl ${ }^{2, b}$ \\ ${ }^{1}$ University of Applied Sciences Heilbronn, Max-Planck-Straße 39, 74081 Heilbronn, Germany \\ ${ }^{2}$ ZTWB Radiochemie München, Technical University of Munich, Walther-Meißner-Str. 3, 85748 \\ Garching, Germany \\ aoliver.kalthoff@hs-heilbronn.de, b'thomas.buecherl@tum.de
}

Keywords: Fission Neutron Tomography, Image Registration, Rotation Axis

\begin{abstract}
In tomography, a misalignment of the rotational axis can introduce blurring. We present intensity-based image registration to calculate the axis' components in three-dimensional space. We have shown that the axis can be deduced from rotating and translating image pairs acquired at $0^{\circ}$ and $180^{\circ}$. No prior experimental calibration or any a-priori knowledge about the system's mechanical setup is necessary. Three samples of different symmetry and homogeneity were examined to experimentally assess the numerical effects of image registration.
\end{abstract}

\section{Introduction}

In fission neutron tomography the reconstruction quality is affected by the correct alignment of the object and the rotational positioning system relative to the detection system. A lateral shift and tilt of the system's rotation axis (SRA) may cause reconstruction algorithms to obscure or simulate image details. Hence for accurate reconstructions, the spatial location of the SRA is crucial. This especially holds for high throughput experiments or in situations where high image contrast is important.

Various methods have been used to account for the tilting of the SRA. A widely used approach is to use a pair of mirrored radiographs taken from complementary viewing angles, for instance at $0^{\circ}$ and $180^{\circ}$. One radiograph is rotated in the detector plane until a similarity measure is optimized with respect to its complement. The rotation angle then represents the tilting of the SRA. A more sophisticated approach has been suggested by [1]. Here, the covariance matrix of an averaged radiograph is calculated from which the (orthonormal) eigenvectors are derived. The rotation axis is identical to the eigenvector corresponding to the largest eigenvalue.

Both approaches make two simplifying assumptions, which do not hold in general. First, a lateral shift of the SRA is assumed to be negligibly small implying that the object's axis is linearly aligned with the SRA. Second, the SRA is supposed to be completely located in the detector plane i.e. perpendicular to the neutron flux.

At TUM's NECTAR facility [2] three samples were investigated with the goal to analytically calculate the SRA's position in three-dimensional space. Since the SRA may be highly sensitive to a particular experimental setup, its position had to be inferable solely from particular radiographs without prior calibration or separate measurements.

The problem has occurred in other fields like X-ray transmission tomography, or X-ray diffraction tomography as well. Gürsoy [3] and earlier Donath [4] assessed artifacts in the reconstructed images, which are evaluated using an optimization framework. Here, the SRA's center is regarded as a parameter, which is iteratively adjusted such that the image's entropy is minimized. Others [5,6] employed feature-based image registration to estimate the lateral shift and tilt of the SRA. Both approaches yield the SRA's projection in the detector plane. 
To the best of our knowledge there is no method to calculate the SRA in three-dimensional space from two-dimensional projections. To achieve this goal, we combined intensity-based image registration with general rotations in three dimensions: While image registration yields the object's planar tilting angle and its translation vector, these observables can be used to calculate the SRA's components in three-dimensional space.

The results of the new method are encouraging and show that the quality of the tomographic reconstruction is be enhanced if the SRA is deduced from the measurement itself. We have shown that the approach is self-contained allowing for the re-assessment of completed measurements.

\section{Methods}

The golden ratio scheme [11] employs a nonsequential decomposition of the sample rotation angle sequence. If the SRA is perfectly adjusted, each radiograph is identical to its mirrored complement. In practice, this condition is difficult to establish. Instead, the image pairs are not aligned or not registered. This effect is best described by a two-dimensional transformation $\tau=\tau(x ; M, \boldsymbol{b})$, where $M$ and $\boldsymbol{b}$ represent a rotation matrix and a translation vector, respectively. Since $M$ and $\boldsymbol{b}$ are not known in advance, $\tau$ has to be deduced from the image pairs. The calculation of $M$ and $\boldsymbol{b}$ is considered an ill-posed problem for which no unique solution exists. Therefore, image registration techniques require a suitable distance measure and a regularizer.

Conversely, $\tau$ can be viewed as a transformation between a reference and a template coordinate system establishing a mapping of corresponding unit vectors (or any linear combination thereof). The same result could have been obtained by a $180^{\circ}$ rotation of unit vectors around the SRA. This rotation is expressed in matrix form from which the SRA's components can be derived.

Image preprocessing. Albeit comprehensive measures to shield the fluroscope detector at the NECTAR facility, any radiograph is subject to imaging noise. This is predominantly reflected in randomly distributed whitish streaks originating from scattered neutrons or secondary gamma radiation. The streaks impose fundamental problems on any image registration technique: Even small disturbances of the input images can cause $M$ and $\boldsymbol{b}$ to become unstable. It is therefore essential to remove the streaks without introducing image blur. A sophisticated method has been suggested by Osterloh et al. [7]. The procedure requires the image's standard deviation as input parameter and operates unattendendly. As a preprocessing step all radiographs were corrected for random streaks.

Image registration. In the following let $R, T \in \mathbb{R}^{m \times n}, m, n \in \mathbb{N}$ be two images acquired at $0^{\circ}$ and $180.43^{\circ}$, respectively ${ }^{1} . R$ is considered as a (fixed) reference, whereas $T$ is a sliding (moving) template [12]. The goal is to find a spatial transformation $\tau(x ; M, b)$ such that $\tau(T ; M, \boldsymbol{b})$ is similar to $R$. Here, similarity is expressed in terms of a distance measure $d \in \mathbb{R}$. The registration problem can then be stated as follows:

Given two images $R$ and $T$ and a distance measure $d$, calculate a transformation $\tau$ such that

$$
d(R, \tau(T ; M, \boldsymbol{b})) \rightarrow \min
$$

The particular choice of $\tau$ depends on the experimental conditions. Three remarks are indicated:

\footnotetext{
${ }^{1}$ Using the golden ratio scan, $T$ cannot be acquired at exactly $180^{\circ}$. Instead, the image closest to $180^{\circ}$ was chosen as template. We considered the angular deviation of $0.43^{\circ}$ to be negligible compared to other imaging defects originating from beam divergence or blurring.
} 
1. Although beam divergence is inherent to any radiographic experiment, its effects are negligible when both the sample's dimensions and the sample-detector distance are small.

2. The detector's exposure time was set large enough to ensure that the neutron flux can be regarded as constant. Therefore, no intensity mapping of image pairs is necessary.

3. Since the samples are rigid bodies, $M \in \mathbb{R}^{2 \times 2}$ is an orthogonal rotation matrix with $\operatorname{det}(M)=1$.

Hence, $\tau(x ; M, \boldsymbol{b})=M x+\boldsymbol{b}$ is a linear or rigid [13] transformation. Several image registration techniques are known to align $R$ and $T$. Intensity based image registration is of special importance since no landmarks (or fiducials) are necessary. A straightforward approach is based on the minimization of the sum of squared differences (SSD, [8] or [9]). In this case (1) is re-written as

$$
d^{S S D}(R, \tau(T ; M, \boldsymbol{b}))=\frac{1}{2}\|R-\tau(T ; M, \boldsymbol{b})\|^{2}=\frac{1}{2} \sum_{i, j}\left(R_{i j}-\tau(T ; M, \boldsymbol{b})_{i j}\right)^{2} \rightarrow \text { min. }
$$

The problem is to find a suitable optimization routine to minimize $d^{S S D}$ with respect to $M$ and b. A Gauss-Newton regularization method was used. This is advantageous, since the sum of squared errors is quadratic in the parameters to be estimated and no Hessian is required [12]. Alternatively, Levenberg-Marquardt techniques have been suggested in the literature [10].

Rotation around the SRA. A rotation of points around an axis through the origin is given by

$$
\left[R_{n}(\alpha)\right]_{i j}=(1-\cos \alpha) n_{i} n_{j}+\cos \alpha \delta_{i j}+\sin \alpha \varepsilon_{i j k} n_{k}
$$

where $\boldsymbol{n}=\left(n_{1}, n_{2}, n_{3}\right)^{T}$ is a unit vector pointing into the axis' direction, $\delta_{i j}$ is Kronecker's Delta and $\varepsilon_{i j k}$ is the Levi-Civita symbol. In our case $\alpha=180^{\circ}$, therfore (3) simplifies to

$$
R_{\boldsymbol{n}}\left(180^{\circ}\right)=\left(\begin{array}{ccc}
2 n_{1}^{2}-1 & 2 n_{1} n_{2} & 2 n_{1} n_{3} \\
2 n_{2} n_{1} & 2 n_{2}^{2}-1 & 2 n_{2} n_{3} \\
2 n_{3} n_{1} & 2 n_{3} n_{2} & 2 n_{3}^{2}-1
\end{array}\right)
$$

Applying (4) to a unit vector of the template frame yields the corresponding unit vector of the reference frame:

$$
\boldsymbol{x}_{R}=R_{\boldsymbol{n}}\left(180^{\circ}\right) \boldsymbol{x}_{T}
$$

where $\boldsymbol{x}_{R}$ is calculated with respect to $M$ and $\boldsymbol{b}$ and $\boldsymbol{x}_{T}$ is an arbitrary vector with $\left\|x_{T}\right\|=1$. Note that only $n_{1}$ and $n_{2}$ can be calculated from (5). Using spherical coordinates one has

$$
\left(\begin{array}{l}
n_{1} \\
n_{2} \\
n_{3}
\end{array}\right)=\left(\begin{array}{c}
\sin \theta \cos \phi \\
\sin \theta \sin \phi \\
\cos \theta
\end{array}\right)
$$

where $\theta$ and $\phi$ are the polar and azimuthal angles, respectively.

Samples. Three samples of different geometrical shape and homogeneity were chosen to assess the results of the intensity-based registration method: a cylindrically shaped object made of plastic, a turbine blade and a plastic bag randomly filled with electronics components and metal parts. While the first and the second sample exhibit well defined contrasts, the third sample appears blurred and inhomogeneous. For each sample the detector's exposure time was 60s. 
Each radiograph was corrected for the dark-current (DC) and open-beam (OB) images according to

$$
x_{c o r r}=\frac{x-x_{D C}}{x_{O B}-x_{D C}} .
$$

\section{Results}

For all samples intensity-based image registration was applied to the image pairs acquired at $0^{\circ}$ $(R)$ and $180^{\circ}(T)$. The results are depicted as superimposed images, where $T$ has been shifted and rotated. The following numeric results were obtained:

$$
\begin{gathered}
M_{\text {cyl }}=\left(\begin{array}{cc}
0.9997 & 0.0232 \\
-0.0232 & 0.9997
\end{array}\right), \quad \boldsymbol{b}_{\text {cyl }}=\left(\begin{array}{c}
38 \\
-9
\end{array}\right) \\
M_{\text {blade }}=\left(\begin{array}{cc}
0.9998 & 0.0199 \\
-0.0199 & 0.9998
\end{array}\right), \quad \boldsymbol{b}_{\text {blade }}=\left(\begin{array}{c}
21 \\
-11
\end{array}\right) \\
M_{\text {bag }}=\left(\begin{array}{cc}
0.9998 & 0.0178 \\
-0.0178 & 0.9998
\end{array}\right), \quad \boldsymbol{b}_{\text {bag }}=\left(\begin{array}{c}
-45 \\
-13
\end{array}\right)
\end{gathered}
$$

The components of $\boldsymbol{b}$ are given in pixels and are rounded. The polar and azimuthal angles for the samples are (c.f. (6))

$$
\theta_{c y l}=0.6^{\circ}, \phi_{c y l}=89.3^{\circ} \quad \theta_{\text {blade }}=0.6^{\circ}, \phi_{\text {blade }}=89.4^{\circ} \theta_{\text {bag }}=0.6^{\circ}, \phi_{\text {bag }}=89.5^{\circ}
$$

Note that, depending on the direction of the coordinate axes, the values of $\theta$ and $\phi$ have to be interpreted accordingly.
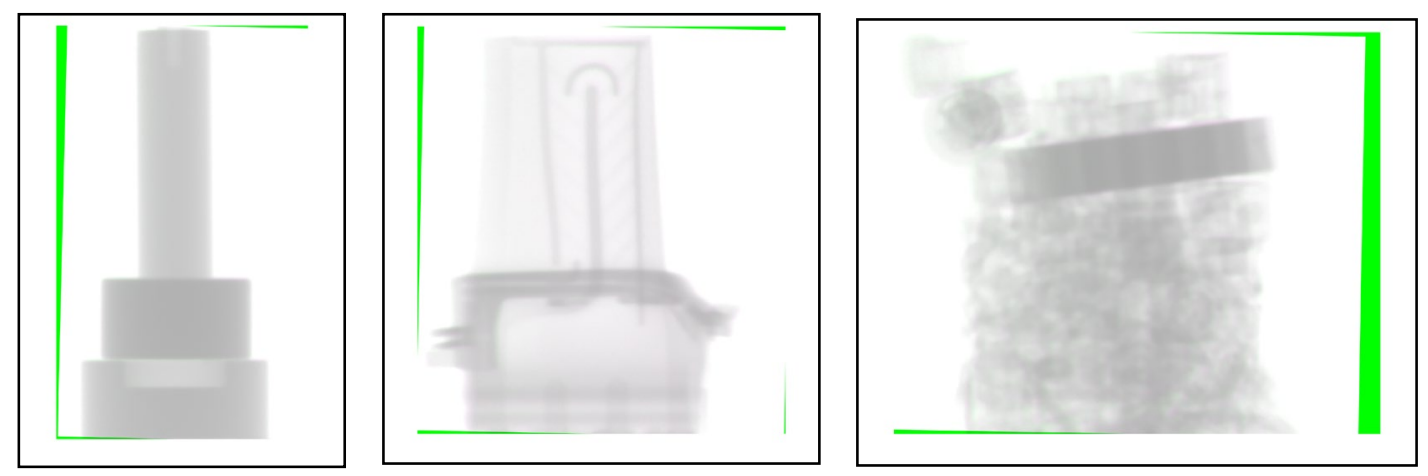

Fig1: Results for mirrored and registrated image pairs. Left: cylinder, middle: turbine blade, right: bag filled with electronics components. The images are displayed in false colors to emphasize the registration effects of rotating and shifting the template images T. Images were cropped and scaled for display.

\section{Discussion}

The goal of estimating the SRA from image pairs was achieved. We presented an analytical method to calculate the SRA's components using intensity-based image registration methods and rotation matrices in three-dimensional space. Comparable results were obtained for the rotation matrix, indicating that the SRA is tilted as expected. Since the rotational device was not modified, the SRA is almost identical for all three samples. The SRA's polar angle $\left(0.6^{\circ}\right)$ is in accordance with the empirical value assumed at NECTAR $\left(0.7^{\circ}\right)$. However, the method yielded a different translation vector for the plastic bag. There are two reasons. First, the plastic bag is 
amorphous and does not exhibit high-contrast structures which are needed for intensity-based registration. Second, image registration is regarded as an ill-posed problem where no unique solution exists. There may be a large number of "local" solutions near the "global" i.e. true minimum of the distance measure. To cure this, manually selected fiducials (or landmarks) can reduce the plurality of solutions. This is in contrast to our intention: The SRA's position had to be allocatable from particular radiographs without prior calibration or additional measurements.

The rotation matrix $M$ and the translation vector $\boldsymbol{b}$ are estimated simultaneously. However, $\boldsymbol{b}$ may be prone to large systematic errors. Recall that the template (or moving) image has to be mirrored prior to image registration. The more the SRA is shifted from the image center, the larger the systematic error in $\boldsymbol{b}$ is: Mirroring anticipates a lateral shift of the template relative to the reference image. Therefore, the template has to be mirrored without a lateral shift. A possible solution is currently being investigated.

It was assumed throughout the experiments, that beam divergence is negligible. If this condition does not hold, image scaling becomes increasingly important. The registration model is still linear, but not any longer rigid in a strict sense. The transformation $M$ is affine and $\operatorname{det}(M)>$ 0 . The problem is aggravated, if scaling and shearing is necessary. This aspect will be assessed for large radioactive waste drums.

Our approach is not restricted to the golden ratio scheme. It can be applied to any acquisition technique as long as the angular difference of the image pairs is (approximately) $180^{\circ}$.

A cautionary note has to be made. Any attempt to retrospectively estimate the SRA from a series of radiographs would be pointless if the true SRA would have been known a priori. Vice versa, it is speculative to assess the correctness of any approach a posteriori if the true SRA is unknown. It would be illustrative to actually compare tomographic reconstruction results based on different methods to estimate the SRA. We feel that this would fit into a review paper.

\section{References}

[1] Z. Ji et al., Calibration and Correction Method of the Deflection Angle of Rotation Axis Projection On Neutron Tomography, Physics Procedia 88 (2017) 299-305.

https://doi.org/10.1016/j.phpro.2017.06.041

[2] Heinz Maier-Leibnitz Zentrum et al., NECTAR: Radiography and tomography station using fission neutrons, Journal of large-scale research facilities 1 (2015) A19. https://doi.org/10.17815/jlsrf-1-45

[3] D. Gürsoy et al., TomoPy: a framework for the analysis of synchrotron tomographic data, J. Synchrotron Rad. 21 (2014) 1188-1193. https://doi.org/10.1107/S1600577514013939

[4] T. Donath, F. Beckmann, A. Schreyer, Automated determination of the center of rotation in tomography data, J. Opt. Soc. Am. A 23(5) (2006) 1048-1057. https://doi.org/10.1364/JOSAA.23.001048

[5] Y. Yang et al., Registration of the rotation axis in X-ray tomography, J. Synchrotron Rad. 22 (2015), 452-457. https://doi.org/10.1107/S160057751402726X

[6] N. Vo, M. Drakopoulos, R.C. Atwood, C. Reinhard, Reliable method for calculating the center of rotation in parallel-beam tomography, Opt. Express 22 (2014) 19078-19086. https://doi.org/10.1364/OE.22.019078

[7] K. Osterloh, T. Bücherl, U. Zscherpel, U. Ewert, Image recovery by removing stochastic artefacts identified as local asymmetries, Journal of Instrumentation 7(4) (2012) C04018.

https://doi.org/10.1088/1748-0221/7/04/C04018 
[8] L.G. Brown, A survey of image registration techniques, ACM Computing Surveys 24(4), 325-376. https://doi.org/10.1145/146370.146374

[9] M. Capek, Optimisation strategies applied to global similarity based image registration methods, WSCG '99: $7^{\text {th }}$ International Conference in Central Europe on Computer Graphics (1999) 369-374.

[10]P. Thévenaz, U.E. Ruttimann, M. Unser, A pyramid approach to subpixel registration based on intensity, IEEE Trans. Image Processing 7(1) (1998) 27-41. https://doi.org/10.1109/83.650848

[11] T. Köhler, A projection access scheme for iterative reconstruction based on the golden section, IEEE Symposium Conference Record Nuclear Science 6 (2004), 3961-3965

[12] J. Modersitzky, Numerical methods for image registration, Oxford University Press on Demand, 2004. https://doi.org/10.1093/acprof:oso/9780198528418.003.0008

[13] J. Modersitzky, FAIR: flexible algorithms for image registration, SIAM (2009). https://doi.org/10.1137/1.9780898718843 